tension in premature infants. J. Pediat., 69: 449 (1966).

17. Thibeault, D. W., Poblete, E., and Auld, P. A. M.: Arteriat-alveolar oxygen difference in premature infants breathing 100\% oxygen. J. Pediat., 71: 814 (1967).

18. Tori, C. A., Krauss, A. N., and Auld, P. A. M.: Serial studies of lung volume and VA/Q in hyaline membrane disease. Pediat. Res., 7: 82 (1973).

19. Dr. A. N. Krauss has a fellowship with the Tuberculosis and Respiratory Disease

Copyright (C) 1976 International Pediatric Research Foundation, Inc.
Association of New York

20. Dr. P. A. M. Auld was supported by United States Public Health Service Research Grant HD-02644.

21. Requests for reprints should be addressed to: P. A. M. Auld, M.D., Division of Newborn Medicine, Department of Pediatrics, New York Hospital Cornell University Medical Center, 522 E. 68th St., New York, N.Y. 10021 (USA). 22. Accepted for publication March 3,1976.

\title{
Skin Reflectance in the Newborn Infant
}

\author{
ALFRED N. KRAUSS, ${ }^{\prime 10}$ PETER W. POST, STEPHANIE WALDMAN, AND PETER A. M. AULD \\ Department of Pediatrics, Division of Perinatology. The New York Hospital-Cornell Medical Center, New York, \\ New York, and Department of Anthropology, The Ohio State University, Columbus, Ohio, USA
}

\section{Extract}

Skin reflectance in red, green, and blue light was measured at the sternum of 99 Caucasian infants ranging in gestational age from 26 to 44 weeks. Skin reflectance was consistently higher in female infants, but this difference was not statistically significant. Highly significant $(P<\mathbf{0 . 0 0 1})$ increases in reflectance at all wave lengths were found when sternal reflectance during the first $\mathbf{4 8} \mathrm{hr}$ of life was related to gestational age. The dispersion of data points about the regression line does not permit this method to be relied upon as the sole means of determining gestational age of infants. Serial studies indicate that reflectance increases in premature infants not given phototherapy, whereas premature infants receiving phototherapy show a fall in reflectance for the duration of therapy. This suggests that phototherapy may cause tanning of the skin.

\section{Speculation}

Reflectance spectrometry provides a safe, noninvasive method to assess human newborn skin maturation and may also be a means to determine effects of various therapeutic regimens on the skin.

The most commonly used system for assessing gestational age of newborn infants is that of Dubowitz and colleagues (3). Many of the items contained in the Dubowitz scale require a subjective evaluation of a particular characteristic on the part of the examiner. Evaluation of the skin for color, thickness, and opacity is required to complete this scale and determine the infant's score. Reflectance spectrophotometry is a safe, noninvasive method for assessing the physical characteristics of the skin and has been widely used and standardized in adults (6). Reflectance of light by the skin depends on the wave length of light used, the skin thickness, and the amount of pigment in the skin. In adults these factors are also influenced by the sex and site of sampling in an individual subject because of variations in the amount of pigment because of sex, relation to pubertal status, and tanning. The aim of the present study was to determine whether measurements of skin reflectance in newborn infants could be standardized to provide a measurement of gestational age and give an objective verification of Dubowitz' data.

\section{METHODS}

Forty-seven male and fifty-two female Caucasian infants were included in this study. Racial identification was voluntarily provided by the parents for birth certificates. All infants were healthy at the time of study and none had received phototherapy before the study. All infants had parents who lived within a 70-mile radius of New York City. The infants ranged in gestational age from 25 to 44 weeks, based on information obtained from the date of the mother's last menstrual period. Additionally, all infants were examined by one of the investigators in order to determine gestational age by means of the Dubowitz criteria (3). If the discrepancy between the mother's dates and physical examination exceeded 2 weeks the infant was excluded from the study. All infants were studied with their parents' knowledge and informed consent according to a protocol approved by the New York Hospital Human Rights Committee.

Infants born after 36 weeks of gestation were studied only once within $48 \mathrm{hr}$ of birth. Premature infants were studied initially within $48 \mathrm{hr}$ of birth and again at 3-day intervals until 1 week of age, and then at weekly intervals until discharge. No mature infant who received phototherapy is included in this study. Some premature infants did receive phototherapy for hyperbilirubinemia, and are so indicated in the appropriate figure. Standard statistical techniques (Student's $t$-test, least squares determination of regression coefficients) were used to compare data between various groups of infants.

Skin reflectance was measured with a model 670 Photovolt "reflection meter" (9). Light from a clear 6-W tungsten bulb is transmitted to the skin at a $0^{\circ}$ angle through various filters and monitored with a compensating photocell (9). Light reflected from the skin is measured by a second photocell at a $45^{\circ}$ angle to the incident beam. Bulb, filters, and photocells form a reflectance unit which is placed directly in contact with the skin. The reflectance unit is connected to a microammeter which records the current generated on a linear scale. The machine is standardized so that full scale current (100\% reflectance) corresponds to the reflection from a magnesium oxide standard tile. High reflectance corresponds to "whiter" skin. Filters used to transmit light were standard Corning glass filters: blue (CS5-60/5543), green (CS4-64/4010), and red (CS2-59/2404). These filters were 2.0, 3.0, and $4.0 \mathrm{~mm}$ thick, respectively. Measurements were made by one 
of the investigators holding the reflectance unit against the skin and reading the instrument's scale to the nearest digit. Because of difficulties in applying the reflectance unit to the forehead and inner upper arm of small infants (standard sites for the investigation of adult populations with this technique), the sternum at the level of the xiphoid was selected as a standard site for application of the reflectance unit. The reflectance for an individual infant represents the average of five determinations with each filter at this site. The average value obtained for an infant had a standard deviation of 1 point of reflectance, and a coefficient of variation of from $2 \%$ for the red filter to $5 \%$ for the blue and green filters. All skin surfaces were clean and dry when studied, the infants having been washed with mild soap as part of routine nursery care.

\section{RESULTS}

Sex is known to affect reflectance in adults. Table 1 compares the sternal reflectance of male and female infants less than $48 \mathrm{hr}$ old and between 35 and 42 weeks of gestational age measured with a red filter. No statistically significant differences were found between male and female infants, although the mean reflectance for female infants was consistently higher than that of males. Since no significant differences were found between male and female infants, data for both sexes was combined throughout the remainder of this report.

Figures 1 to 3 depict the variation in the sternal reflectance of Caucasian infants, measured with red, blue, and green filters, during the first $48 \mathrm{hr}$ of life and before the institution of phototherapy at gestational ages ranging from 25 to 44 weeks. When skin reflectance was correlated with gestational age, the highest correlation coefficient $(r)$ was found when a linear equation was used to describe the data. Increasing reflectance was observed with all three filters as gestational age of the subject increased. Statistically, correlation between skin reflectance and gestational age was good $(r=0.56-0.78)$ and significantly different from $0(P$ $<0.001$ )

Serial studies of sternal reflectance of red light in premature infants are depicted in Figure 4. Data points obtained from infants not receiving phototherapy are connected with solid lines (-). Those obtained from infants receiving phototherapy are connected by broken lines (- - ). The points are plotted about the regression line for data obtained during the first $48 \mathrm{hr}$ of life, and the $95 \%$ ranges about the mean values of sternal reflectance in mature infants are also included for reference. Serial measurements of reflectance in red light indicate that a fall in sternal reflectance takes place during phototherapy in seven of nine infants receiving this treatment. In contrast, the reflectance in most infants not receiving phototherapy increases at a slope similar to that of the regression line and approaches the mean values of sternal reflectance in mature infants.

\section{DISCUSSION}

The present study confirms the previous work of Dubowitz et al. (3), suggesting that the appearance of the skin in Caucasian infants

Table 1. Sternal reflectance values $(R)$ of Caucasian male and female infants less than $48 \mathrm{hr}$ of age and measured with red light ${ }^{1}$

\begin{tabular}{|c|c|c|c|c|c|c|}
\hline \multirow{2}{*}{$\begin{array}{c}\text { Gestational } \\
\text { age, } \\
\text { weeks }\end{array}$} & \multicolumn{2}{|c|}{ Male infants } & \multicolumn{2}{|c|}{ Female infants } & \multirow[b]{2}{*}{$t$} & \multirow[b]{2}{*}{$P$} \\
\hline & $R$ & $n$ & $R$ & $n$ & & \\
\hline $35-36$ & $57.1 \pm 1.9$ & 3 & $57.3 \pm 4.9$ & 5 & 0.0705 & NS \\
\hline $37-38$ & $58.0 \pm 2.1$ & 6 & $58.4 \pm 2.1$ & 5 & 0.2813 & NS \\
\hline $39-40$ & $58.9 \pm 2.5$ & 13 & $59.5 \pm 2.1$ & 13 & 0.7234 & NS \\
\hline $41-42$ & $59.0 \pm 1.2$ & 5 & $60.1 \pm 1.7$ & 8 & 1.2011 & NS \\
\hline
\end{tabular}

${ }^{1}$ Reflectance values are population mean values \pm 1 SD. NS: not significant.

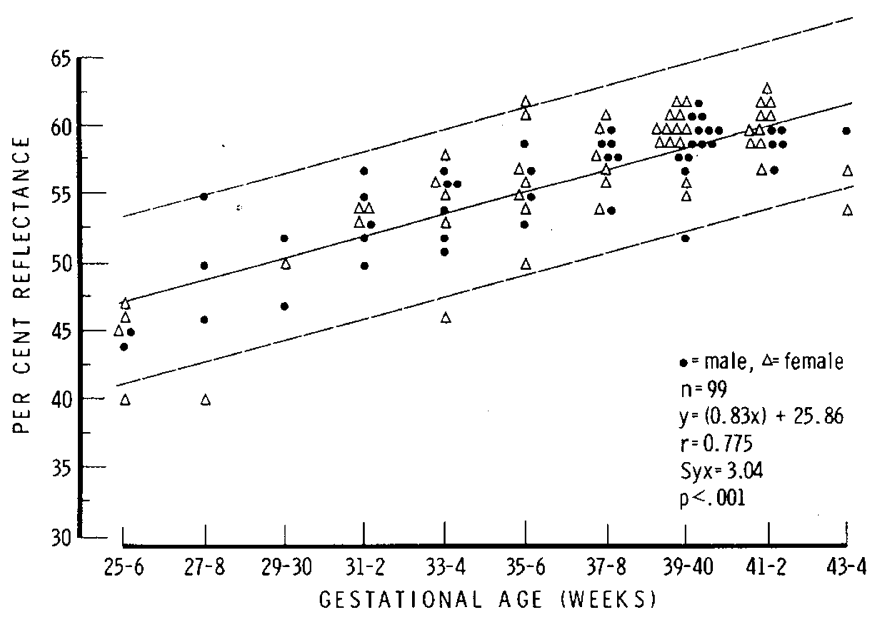

Fig. 1. Sternal reflectance (percent) with red filter of Caucasian infants plotted against gestational age. All measurements taken within the first 48 $\mathrm{hr}$ of life. _ in the regression, _... represent 2 SD and include $95 \%$ of the data.

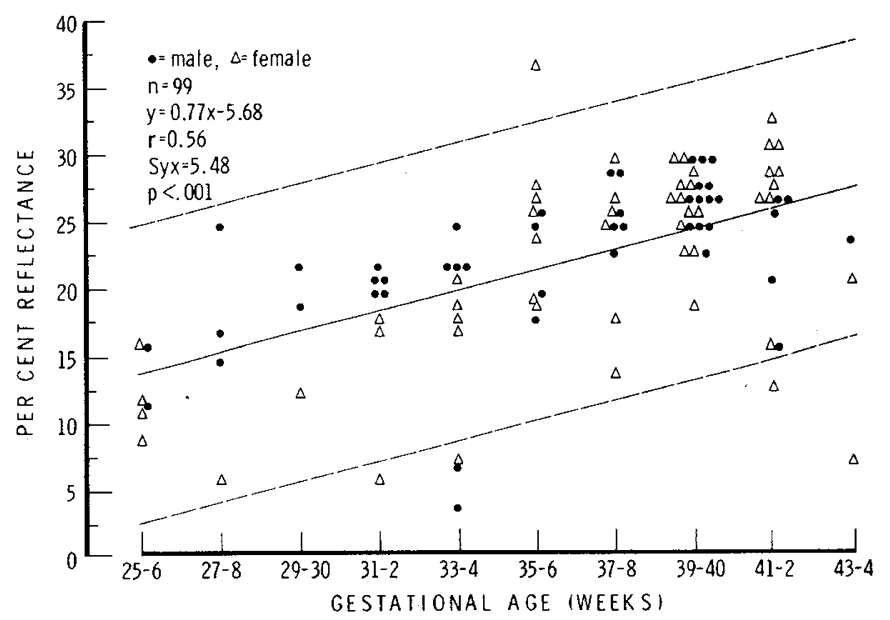

Fig. 2. As Figure 1, but measured with a green filter.

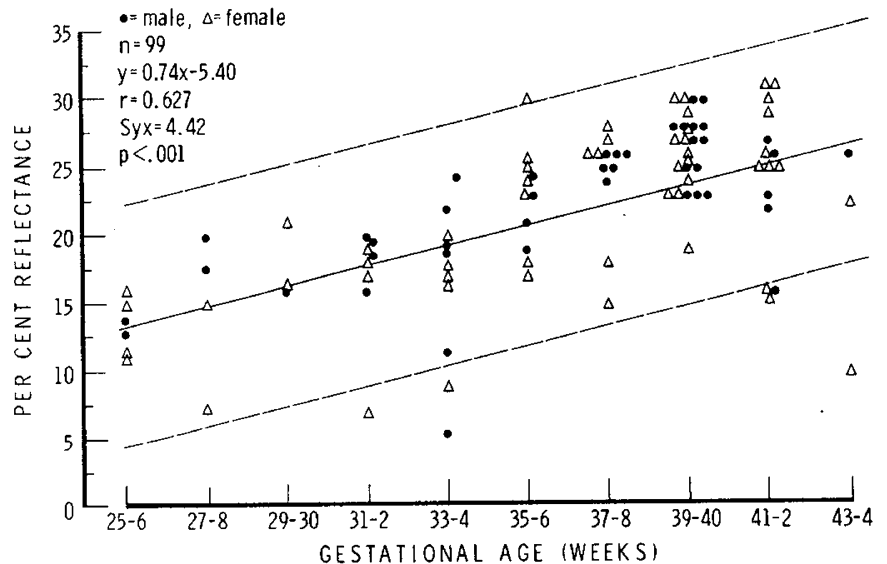

Fig. 3. As Figure 1, but measured with a blue filter.

changes significantly with increasing gestational age. Ballowitz and Avery (1), using continuous spectral reflectance to describe the characteristics of the human skin, found results similar to those described in the present study. They noted that reflectance in the blue and green portions of the spectrum was greatly reduced compared with reflectance in red light. The present report extends their results to a large group of newborns ranging between 25 and 44 weeks of gestational age. Reflectance with the red filter is thought to measure primarily melanin, whereas reflectance with 


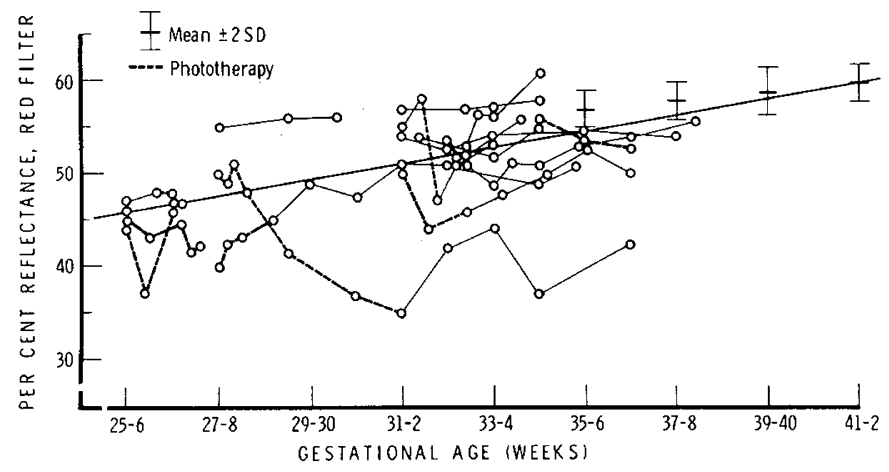

Fig. 4. Serial determinations of sternal skin reflectance obtained with a red filter on a group of premature Caucasian infants. Data points for individual infants are connected. Those receiving phototherapy are connected with - - - . The regression line determined from data presented in Figure 1 and mean reflectance values $\pm 2 \mathrm{SD}$ for infants of $35-42$ weeks of gestational age is also represented.

the green filter is related primarily to yellow pigments such as carotene: reflectance with the blue filter is related primarily to bilirubin and hemoglobin $(2,5,6)$.

The thinner, more transparent skin of the immature infant reflects less light than the skin of the gestationally more mature infant. The increase in sternal reflectance in blue and green light with increasing gestational age suggests the possibility of increasing thickness of skin in relation to the amounts of bilirubin and hemoglobin present. These possibilities require further investigation. Data depicted in Figures 1 to 3 demonstrate that reflectance of the skin, measured within the first $48 \mathrm{hr}$ of extrauterine life, increases with increasing gestational age. Reflectance spectrophotometry, especially when performed in red light, provides an objective method of assessing gestational age in newborn infants during the first $48 \mathrm{hr}$ of life. Although a good correlation between sternal reflectance and gestational age is obtained, the dispersion of the study population about the mean regression line is large. The method is thus not sufficiently sensitive to differentiate between a very pale infant of $28-30$ weeks of gestation and an average infant of 34-36 weeks of gestation.

Serial studies of sternal reflectance in red light in premature infants, depicted in Figure 4, reveal that sternal reflectance increases during extrauterine life as it does during intrauterine life. Infants not receiving phototherapy, whose data points are connected by solid lines $(-)$ in Figure 4 , have values of sternal reflectance which tend to increase after birth at the same rate as the regression line determined from data obtained during the first $48 \mathrm{hr}$ of extrauterine life and reflecting essentially changes associated with intrauterine development. Reflectance of sternal skin increases in individual premature infants as they grow older, suggesting that the factors which contribute to increasing reflectance during intrauterine life continue their maturation after birth. As indicated by the data points in Figure 4 connected with broken lines (- - ), these processes may be altered by phototherapy.

Woody and Brodkey (8) demonstrated tanning in the skin of a newborn infant receiving phototherapy. Reflectance spectrophotometry would indicate tanning by a fall in reflectance measured in red light. This was observed in seven of the nine infants receiving phototherapy, in conjunction with a subsequent increase in sternal reflectance after discontinuation of phototherapy. Tanning of the skin on exposure to light may result from immediate darkening due to an increase in the proportion of melanized melanosomes and changes in their distribution (immediate pigment darkening), and delayed tanning due to the formation, melanization, and transfer of melanosomes to keratinocytes (4). The immediate pigment darkening begins on exposure to light and fades within 3-4 hr, although it may persist for up to $24 \mathrm{hr}$ (7). Delayed tanning begins $24-72 \mathrm{hr}$ after exposure to light and usually reaches a maximum between 7 and 9 days after exposure (5). The intensity of the response and the rate of fading depends on the duration and amount of energy received by the skin as well as the inherent pigment characteristics of the individual. The early onset of sternal darkening seen in seven of nine infants receiving phototherapy suggests that immediate pigment darkening may occur in premature infants.

\section{SUMMARY}

Measurements of sternal skin reflectance in red, green, and blue light were carried out in 99 Caucasian infants ranging in gestational age from 26 to 44 weeks. Increasing reflectance was found with increasing gestational age. This finding is consistent with conventional methods of dating infants by external physical criteria and is related to the thickness and opacity of the skin. The method is not sufficiently sensitive to provide by itself an accurate objective method of determining the gestational age of a newborn infant. An additional finding was that of darkening of the skin after the institution of phototherapy in infants being treated for hyperbilirubinemia. Reflectance spectrophotometry provides a safe, noninvasive method for assessing the maturation of human newborn skin and may also provide a means of determining the effects of various therapeutic regimens on the skin.

\section{REFERENCES AND NOTES}

1. Ballowitz, L., and Avery, M. E.: Spectral reflectance of the skin. Biol. Neonate, 15: 348 (1970).

2. Daniels, F., and Imbrie, J. D.: Comparison between visual Grading and reflectance measurements of erythema produced by sunlight. J. Invest. Dermatol. 30: 295 (1958).

3. Dubowitz, L. M. S., Dubowitz, V., and Goldberg, C.: Clinical assessment of Gestational age in the newborn infant. J. Pediat., 77: 1 (1970).

4. Jimbow, K., Pathak, M. A., Szabo, G., and Fitzpatrick, T. B.: Ultrastructural changes in human melanocytes after ultraviolet radiation. In: T. B. Fitzpatrick: Sunlight and Man: Normal and Abnormal Photobiologic Responses, p. 195-215 (University of Tokyo Press, Tokyo, 1974).

5. Lee, M. M. C., and Laker, G. W.: The suntanning potential of human skin. Human Biol., 31: 252 (1959).

6. Tregar, R. T.: Physical Functions of the Skin, p. 96-113 (Academic Press, New York, 1966).

7. Pathak, M. A., and Stratton, K.: Effects of ultraviolet and visible radiation and the production of free radicals in the skin. In: F. Uhrbach: The Biologic Effects of Ultraviolet Radiation (Pergamon Press, New York, 1969).

8. Woody, N. C., and Brodkey, M. J.: Tanning from phototherapy for neonatal jaundice. J. Pediat., 82: 1042 (1973).

9. Photovolt Corporation, New York, N.Y.

10. Requests for reprints should be addressed to: A. N. Krauss, M.D., Department of Pediatrics, Division of Perinatology, New York Hospital-Cornell Medical Center, 1300 York Ave., New York, N. Y. 10021 (USA).

11. Accepted for publication April 1, 1976. 